\title{
Ellagic acid alleviates high glucose-induced podocyte and renal epithelial cell apoptosis and hyperglycemia-induced renal injury by regulating NF-KB/miR-150-3p/BCL2
}

\section{Ying Cai ( $\nabla$ cai_ying1122@sohu.com )}

Lihuili Hospital https://orcid.org/0000-0002-7516-3934

\section{Yong Xu}

Medicalsystem Biotechnology Co., Ltd.,

\section{Qicheng Ni}

Shanghai Jiaotong University: Shanghai Jiao Tong University

\section{Bei Guo}

lihuili hospital

\section{Sheng Chen}

lihuili hospital

Xiaoli Jiang

lihuili hospital

Qiyuan Wu

lihuili hospital

\section{Research Article}

Keywords: ellagic acid, podocyte, apoptosis, miR-150-3p, diabetic nephropathy

Posted Date: April 30th, 2021

DOI: https://doi.org/10.21203/rs.3.rs-468966/v1

License: (c) (i) This work is licensed under a Creative Commons Attribution 4.0 International License. Read Full License 


\section{Abstract \\ Objective}

Ellagic acid (EA) as a multi-target bioactive compound has been reported to improve diabetes-related complications, including diabetic nephropathy (DN). Herein, we plan to investigate the molecular mechanism underlying EA-mediated renal protection in diabetic mice.

\section{Methods}

Streptozotocin (STZ; $35 \mathrm{mg} / \mathrm{kg}$ successive injection for 5 times) was applied to establish DN model in mice. Normal or diabetic mice were administrated by EA ( $100 \mathrm{mg} / \mathrm{kg} /$ day $)$ by intragastric administration for 8 weeks. In vitro diabetic cell model, podocytes and renal tubular epithelial cells (RTECs) were exposed to normal glucose (NG; $5 \mathrm{mM}$ ) or high glucose (HG; $30 \mathrm{mM})$.

\section{Results}

Our results demonstrated that EA treatment prevented HG-induced podocyte and RTEC apoptosis and growth inhibition by inhibiting NF-KB/miR-150-3p to activate BCL2 in vitro. In vivo diabetic model of mice, EA administration improved renal filtration function, tubular and glomerular injury, and interstitial fibrosis. More importantly, supplementation of EA also suppressed NF-KB/miR-150-3p activation and accelerated BCL2 expression in the kidney of diabetic mice. In another experiment, miR-150-3p antagomir as a potential gene therapeutic choice has been validated to rescue hyperglycemia-induced renal dysfunction in mouse model. Taken together, in vitro and in vivo experimental measurements corroborate that EA modulates NF-KB/miR-150-3p/BCL2 cascade signaling to attenuate renal damage in diabetic models.

\section{Conclusion}

Our findings revealed that EA modulated the suppression of NF-KB/miR-150-3p to activate BCL2 that contributed to prevent hyperglycemia-induced renal dysfunction. In addition, synthetic miR-150-3p antagomir or inhibitors could alleviate tubular injury and interstitial fibrosis, and prevent HG-induced podocyte and RTEC apoptosis.

\section{Introduction}

Podocyte and renal tubule epithelial cell apoptosis have frequently been reported in diabetic nephropathy (DN) and contribute to glomerular dysfunction and tubular atrophy that are the most prominent characteristics of kidney damage to deteriorate renal reabsorption and filtration [1-3]. Therefore, the prevention of podocyte and tubule epithelial cell apoptosis may present prospective therapeutic strategies for preventing diabetic kidney diseases. 
Ellagic acid (EA), as a dimeric derivative of gallic acid, is the major hydrolysate of ellagitannins and is extensively excavated in natural nutriments [4]. At present, EA is recognized as a multi-target bioactive compound to improve health problems, including cardiovascular diseases, dyslipidemia, obesity, neurodegenerative disorders and cancers [4-8]. In addition, EA exhibits potential protective action towards diabetes-related complications, including DN [9-11]. Mechanically, EA mediates multiple signaling pathways, such as nuclear factor-kappa B (NF-KB), inflammatory signaling and non-coding RNA-modulated keap1/Nrf2 system [9, 10, 12].

microRNAs (miRs) are belonged to non-coding RNAs and are implicated in numerous physio-pathological progression [13-15]. miRs are responsible for the post-transcriptional repression of protein translation by binding with the $3^{\prime}$-untranslated regions (3'-UTRs) of target genes [14]. Increasing studies corroborate that abnormal miRs (miR-15b-5p, miR-133b, miR-200 and miR-320a) attribute to hyperglycemia-induced renal injury [16-19], suggesting that miR-related therapeutic drugs may become imperative therapeutic options for DN. Previous studies have indicated that EA modulates miR-223, miR-140-3p to attenuate insulin resistance and acute myocardial infarction $[12,20]$. Herein, we aim to determine whether EA-regulated miR-150-3p is involved in HG-induced podocyte and renal epithelial cell apoptosis and hyperglycemiainduced renal injury.

\section{Material And Methods}

Animal model. C57BL/6J mice (6-8 week-old; $20 \pm 2 \mathrm{~g} ; \mathrm{n}=48$ ) were obtained from Shanghai SLAC Laboratory Animal Co., Ltd., Shanghai, China. After one week of adaptive feeding, streptozotocin (STZ; 35 $\mathrm{mg} / \mathrm{kg}$ successive injection for 5 times within one week) was used to establish a mouse model with diabetes mellitus. Normal and STZ-injected diabetic mice were classified into four groups $(\mathrm{n}=6$ in each group) as follows: (1) Control (Con) group with normal saline (0.9\%); (2) Con + EA group with EA (100 $\mathrm{mg} / \mathrm{kg} /$ day; Sigma-Aldrich) treatment; (3) STZ group with normal saline (0.9\%); (4) STZ + EA group with EA $(100 \mathrm{mg} / \mathrm{kg} /$ day) treatment. In another experiment, mice were classified into four groups $(\mathrm{n}=6$ in each group) as follows: (1) Con group; (2) STZ group; (3) STZ + anti-Con; (4) STZ + anti-miR-150-3p (miR-150$3 p$ antagomir; $20 \mathrm{nM} / 0.1 \mathrm{~mL}$; once/two weeks for 8 weeks by tail-vein injection). miR-150-3p antagomir and anti-Con were synthesized by Guangzhou RiBo Biotech Co., Ltd., Guangzhou, China. Animal feeding, anesthesia, sacrifice and specimen collection were performed according to ARRIVE guidelines 2.0 [21]. The animal experiment was approved by the Ethics Committee of the Lihuili Hospital.

Cell culture. Mouse podocyte and renal tubular epithelial cell (RTEC) were obtained from ATCC and cultured with DMEM/F-12 medium (Gibco) with $10 \%$ fetal bovine, $95 \%$ air, $5 \% \mathrm{CO}_{2}$ at $37{ }^{\circ} \mathrm{C}$. EA $(0-30 \mu \mathrm{M})$ was dissolved in $10 \%$ dimethyl sulfoxide to stimulate normal or $\mathrm{HG}(30 \mathrm{mM})$-treated podocytes and RTECs.

Cell transfection. miR-150-3p mimics (5'-CUGGUACAGGCCUGGGGGAUAG-3') and inhibitors (5'CUAUCCCCCAGGCCUGUACCAG-3') were synthesized by RiBo Biotech (Guangzhou, China) and 
transfected into podocytes and RTECs using Lipofectamine 2000 (Invitrogen), according to the manufacturer's protocols.

Cell counting kit 8 (CCK8). After podocytes or RTECs were treated with EA, HG or miR-150-3p inhibitors, cell viability was evaluated by CCK8 kits (Dojindo, Japan) according to the manufacturer's instructions. The absorbance was measured at $450 \mathrm{~nm}$ with a SpectraMax M5 ELISA plate reader (Molecular Devices, LLC, Sunnyvale, CA, USA).

TUNEL assay. Cell apoptosis detection TUNEL kit (Roche) was used to analyze apoptotic cells with TUNEL positive staining (red staining) with fluorescence microscopy according to the manufacturer's instructions.

Luciferase reporter assay. Podocytes or RTECs $\left(1 \times 10^{5}\right)$, containing BCL2 wild-type (WT) or mutant-type (Mut) 3'-UTR (Sangon Biotech, Shanghai, China), were transfected with miR-150-3p mimics or miR-Con, and then luciferase activity was evaluated with a dual-luciferase reporter assay kit (Beyotime, Haimen, China).

Western blot. Standard immunoblotting was performed as described previously [22]. NF-KB/p65 (Cell Signaling Technology; cat. no: \#59674), B cell leukemia/lymphoma 2 (BCL2) (Abcam; cat. no: ab117115), fibronectin 1 (FN1) (Santa Cruz Biotech; cat. no: sc-59826) and nephrin (Abcam; cat. no: ab216692) primary antibodies were used to incubate protein membrane. Histone (Cell Signaling Technology; cat.no: \#9715) and $\beta$-actin (Abcam; cat. no: ab179467). signals were used as an internal reference. Protein bands were obtained using an ECL chemiluminescence kit (Santa Cruz Biotech, Santa Cruz, CA, USA) with Bio-Rad Gel Imaging System (Bio-Rad Laboratories, Inc., Hercules, CA, USA). Quantitative data were analyzed using Quantity One® software version 4.5 (Bio-Rad Laboratories, Inc., Hercules, CA, USA).

RT-qPCR. Standard RT-qPCR procedures were performed as described previously [23]. The PCR primers were listed as follows: forward 5'- CTGGTACAGGCCTGGGGGATAG-3' and reverse 5'-

TGGTGTCGTGGAGTCG-3' for miR-150-3p; forward 5'-CTCGCTTCGGCAGCACA-3' and reverse 5'AACGCTTCACGAATTTGCGT-3' for U6; forward 5'-AGCAGTCGGTACAACTTAAAGG-3' and reverse 5'ACTCGACAACAATACAGACCAC-3' for kidney injury molecule 1 (KIM1); forward 5'TGGCCCTGAGTGTCATGTG - 3' and reverse 5'-CTCTTGTAGCTCATAGATGGTGC-3' for neutrophil gelatinase-associated lipocalin (NGAL); forward 5'-ATGGGAGCTAAGGAAGCCACA-3' and reverse 5'CCACACCACAGCTTAACTGTC-3' for nephrin; forward 5'-ATGTGGACCCCTCCTGATAGT-3' and reverse 5'GCCCAGTGATTTCAGCAAAGG-3' for FN1; forward 5'-GCTCCTCTTAGGGGCCACT-3' and reverse 5'ATTGGGGACCCTTAGGCCAT-3' for collagen, type I, alpha 1 (Col1a1); forward 5'-

CTGTAACATGGAAACTGGGGAAA-3' and reverse 5'-CCATAGCTGAACTGAAAACCACC-3' for collagen, type III, alpha 1 (Col3a1); forward 5'-GAAATGCCACCTTTTGACAGTG-3' and reverse 5'-

TGGATGCTCTCATCAGGACAG-3' for interleukin 1 beta (IL-1 $\beta$ ); forward 5'-CTGCAAGAGACTTCCATCCAG-3' and reverse 5'-AGTGGTATAGACAGGTCTGTTGG-3' for IL6; forward 5'-TAAAAACCTGGATCGGAACCAAA-3' 
and reverse 5'-GCATTAGCTTCAGATTTACGGGT-3' for monocyte chemotactic protein 1 (MCP1); forward 5'AGGTCGGTGTGAACGGATTTG-3' and reverse 5'-GGGGTCGTTGATGGCAACA-3' for GAPDH.

Biomarker in serum, urine and tissues. Tumor necrosis factor-a (TNF-a), IL-1 $\beta$, IL-6 (Elabscience Biotechnology Co.,Ltd., Wuhan, China), blood urea nitrogen (BUN), serum creatinine ( $\mathrm{Cr}$ ) and urinary total protein (BIOSINO, Beijing, China), urinary KIM1 and NGAL (Elabscience Biotechnology Co.,Ltd., Wuhan, China) were measured according to the manufacturer's instructions. Alanine aminotransferase (ALT) and aspartate aminotransferase (AST; Nanjing Jiancheng Bioengineering Institue, Nanjing, China) were measured using enzyme-linked immunosorbent assays according to the manufacturer's instructions.

Histologic examination. Hematein \& eosin (H\&E) staining, periodic acid-schiff staining and Masson's Trichrome staining, and the scoring of tubular, glomerular injury and interstitial fibrosis were performed as described previously [24].

Statistical analysis. Data were expressed as mean and standard deviation. Statistical analysis was performed with the GraphPad Prism 7.0 (GraphPad Software, Inc., La Jolla, CA, USA). Inter-group comparisons were calculated using a one-way analysis of variance. $P$ value of less than 0.05 was indicated a significant difference.

\section{Results}

\section{EA attenuates inflammatory response apoptosis in HG-stimulated RTECs and podocytes.}

To determine whether EA protects against HG-induced RTEC and podocyte dysfunction, the cytotoxicity of EA was investigated in RTECs and podocytes exposure to HG $(30 \mathrm{mM})$ for $24 \mathrm{~h}$. As shown in Figure 1A, EA had no obvious inhibitory effect on cell viability when the concentration of EA less than $30 \mu \mathrm{M}$. Compared with NG $(5 \mathrm{mM})$ group, cell viability was significantly inhibited by approximately $40 \%$ in the HG group. However, HG-induced growth inhibition of RTECs and podocytes was rescued by EA treatment (Figure 1A). Inflammatory cytokines, including TNF-a, IL-1 $\beta$ and IL-6, levels were significantly increased by HG stimulation; however, EA treatment sharply down-regulated HG-evoked inflammatory response in RTECs and podocytes (Figure 1B and 1C). We also found that EA treatment reduced the proportion of apoptotic cells in HG-stimulated RTECs and podocytes (Figure 1D).

\section{EA mediates the NF-KB/miR-150-3p axis in HG-induced RTEC and podocyte apoptosis.}

EA has been highlighted as a NF-KB inhibitor to alleviate multiple pathological processes, including DN [9]. Moreover, NF-KB can modulate miR-150-3p expression in several diseases [25, 26]. Herein, we hypothesized that EA-mediated NF-KB/miR-150-3p might be implicated in HG-induced RTEC and podocyte apoptosis. As shown in Figure 2A, 2B and 2C, both nuclear NF-KB protein and miR-150-3p expression levels were significantly elevated in HG-stimulated RTECs and podocytes compared with those cells treated with NG. However, EA treatment inhibited HG-activated NF-KB and miR-150-3p in RTECs and 
podocytes. To further investigate the roles of miR-150-3p in HG-induced RTEC and podocyte apoptosis, miR-150-3p inhibitors were transfected into RTECs and podocytes to silence the expression of miR-150$3 p$. As shown in Figure 2D, transfection of miR-150-3p inhibitors significantly declined miR-150-3p expression in RTECs and podocytes compared with the control group. After transfection with miR-150-3p inhibitors into HG-stimulated RTECs and podocytes, our findings suggested that HG-induced apoptosis (Figure 2E) and growth inhibition (Figure 2F) were rescued by the inhibition of miR-150-3p expression.

\section{BCL2 expression can be regulated by miR-150-3p and EA}

In our study, based on online bioinformatics analysis and luciferase assays, our findings suggested that $B C L 2$ is a direct target of miR-150-3p, reflecting that transfection with miR-150-3p mimics into BCL2 3'UTR-WT podocytes and RTECs significantly reduced luciferase activity, but the luciferase activity had no obvious change in BCL2 3'-UTR-Mut cells (Figure 3A). In addition, overexpression or silencing of miR-150$3 p$ significantly repressed or up-regulated the protein expression of BCL2 in podocytes and RTECs, respectively (Figure 3B). In vitro experimental measurements revealed that HG led to a significant reduction of BCL2 protein expression in podocytes and RTECs, while EA treatment reversed HG-induced BCL2 inhibition (Figure 3C and 3D). These findings indicate that EA-evoked BCL2 activation may be associated with the inhibition of the NF-kB/miR-150-3p axis in vitro.

\section{EA has no effect on body weight and FBG in diabetic mice.}

To further investigate the role of EA on renoprotective action, we performed a pharmacological experiment in diabetic mice with or without EA administration. Physiological parameters, body weight and FBG, exhibited that a significant decrease in body weight and an increase in FBG were observed in the experimental period after STZ injection (Figure 4A and 4B). However, EA administration for 8 weeks had no obvious effect on body weight and FBG in diabetic mice (Figure 4A and 4B).

\section{EA mitigates renal injury in diabetic mice.}

To explore the renal protective activity of EA on hyperglycemia-induced renal dysfunction, STZ mice were administrated with or without EA for 8 weeks. BUN and serum $\mathrm{Cr}$ (Figure 5A), mRNA expression of KIM1 and NGAL (Figure 5B), urinary KIM1 and NGAL (Figure 5C), and urinary total protein (Figure 5D) were significantly elevated in diabetic mice compared with those of normal mice. Podocyte injury marker nephrin mRNA expression was sharply reduced in the kidney of diabetic mice (Figure 5E). Intriguingly, administration of diabetic mice with EA for 8 weeks markedly improved these pathological parameters. Histologic examination by PAS staining and H\&E staining exhibited that tubular atrophy (Figure $5 F$ ) and 
glomerular pyknosis (Figure 5G) were presented in diabetic mice. Histologic scoring indicated that tubular and glomerular injury were significantly attenuated in diabetic mice with EA treatment (Figure $5 \mathbf{F}$ and $\mathbf{5 G}$ ).

\section{EA alleviates interstitial fibrosis in diabetic mice.}

As shown in Figure 6A, there was strong collagen deposition with blue staining in STZ-treated mice. However, the density of blue staining was obviously reduced in diabetic mice with EA administration (Figure 6A). Semiquantitative scoring validated that hyperglycemia-induced interstitial fibrosis in diabetic mice was dramatically mitigated by EA administration (Figure 6B). As shown in Figure 6C, 6D and 6E, upregulation of fibrosis markers FN1, Col1a1 and Col3a1 mRNA expression levels in diabetic mice were significantly reversed by EA administration.

\section{EA mediates NF-KB/miR-150-3p/BCL2 activity in diabetic mice.}

EA-mediated the activation of BCL2 via inhibiting NF-KB/miR-150-3p axis has been observed in HGstimulated podocytes and RTECs. Next, we further explore the effect of EA on NF-KB/miR-150-3p/BCL2 activity in the kidney of STZ-treated mice. We also found that hyperglycemia facilitated nuclear NF-KB protein and miR-150-3p expression and inhibited BCL2 protein expression in the kidney. However, hyperglycemia-induced up-regulation of NF-KB protein (Figure 7A) and miR-150-3p (Figure 7B) and downregulation of BCL2 (Figure 7C) were reversed by EA treatment. In addition, hyperglycemia-induced upregulation of pro-inflammatory cytokines and mediators, IL-1 $\beta$ (Figure 7D), IL-6 (Figure 7E) and MCP1 (Figure 7F), was strikingly reduced by EA administration.

\section{The effect of miR-150-3p antagomir in kidney, heart and liver of diabetic mice.}

To further investigate the role of miR-150-3p in the pathogenesis of DN, diabetic mice were treated by the injection of miR-150-3p antagomir. Compared with diabetic mice, miR-150-3p expression in the kidney, heart and liver was significantly reduced after received with anti-miR-150-3p treatment (Figure 8A). Histologic examination exhibited that anti-miR-150-3p administration markedly improved hyperglycemiainduced tubular and glomerular injury (Figure 8B). However, both STZ and anti-miR-150-3p treatment had no obvious detrimental effect on the morphology of heart and liver (Figure 8B) and serum ALT and AST (Figure $8 \mathrm{C}$ ), suggesting that anti-miR-150-3p treatment had no obvious influence on cardiac and hepatic functions. Collectively, anti-miR-150-3p treatment ensures medication safety in vivo experiments.

\section{Anti-miR-150-3p treatment mitigates renal injury in diabetic mice.}


To explore the function of miR-150-3p antagomir, diabetic mice were received miR-150-3p antagomir treatment by tail intravenous injection biweekly for 8 weeks. As shown in Figure 9A, 9B and 9C, hyperglycemia-induced the up-regulation of renal injury marker, BUN, serum $\mathrm{Cr}$ and $24 \mathrm{~h}$ urinary total protein, was decreased by anti-miR-150-3p injection. Histologic examination revealed that hyperglycemiainduced tubular injury (Figure 9D) and interstitial fibrosis (Figure 9E) were dramatically alleviated by antimiR-150-3p injection. As shown in Figure 9F, fibrotic biomarker FN1 protein expression was significantly reduced in the kidney of diabetic mice after anti-miR-150-3p injection. Both nephrin (Figure 9G) and BCL2 (Figure $9 \mathrm{H}$ ) protein expression was significantly increased in the kidney of diabetic mice after anti-miR150-3p injection. Based on in vitro and in vivo findings, our study deducted that EA performs a renal protective activity, at least partly, by mediating the NF-KB/miR-150-3p/BCL2 signaling axis (Figure 10).

\section{Discussion}

In alternative therapeutic schedules for DN, EA administration displays diversiform protective effects, including the reduction of proteinuria and podocyte loss, the improvement of oxidative stress and inflammatory response in a diabetic animal model [9-11]. In our study, we further investigate the molecular mechanism underlying EA-mediated renal protection in diabetic mice. Our results demonstrated that EA treatment prevented HG-induced podocyte and RTEC apoptosis and growth inhibition by inhibiting NF-KB/miR-150-3p to activate BCL2 in vitro. In vivo diabetic model of mice, EA administration improved renal filtration function, tubular and glomerular injury, and interstitial fibrosis. More importantly, supplementation of EA also suppressed NF-KB/miR-150-3p activation and accelerated BCL2 expression in the kidney of diabetic mice. In another experiment, miR-150-3p antagomir as a potential gene therapeutic choice has been validated to rescue hyperglycemia-induced renal dysfunction in a mouse model. Taken together, in vitro and in vivo experimental measurements corroborate that $E A$ modulates NF-KB/miR-150-3p/BCL2 cascade signaling to attenuate renal damage in diabetic models.

$\mathrm{NF}-\mathrm{KB}$ is the most predominant transcription factor responsible for the transcription of inflammatory cytokines [27]. Accumulative studies indicate that over-activation of nuclear NF-kB is closely connected with hyperglycemia- or high fat-induced sterile inflammatory response in the progression of renal damage $[9,28,29]$. Overexpression of NF-KB is also implicated in various animal models with acute or chronic kidney diseases [30-32]. For example, NF-KB is a central molecule to activate inflammatory response in lipopolysaccharide-induced acute renal damage [30, 31]. Activation of NF-KB/p38-MAPK signaling pathway attributes to renal inflammation in high-fat diet/STZ-induced diabetes in mice [32]. These conclusions manifest that NF-KB represents a valuable therapeutic target to rescue renal injuries. EA has been reported as NF-KB inhibitor to reduce the secretion of pro-inflammatory cytokines and improve renal morphological damage in type II diabetic rats [9]. In the mouse model of STZ-induced DN, the concentration of EA more than $50 \mathrm{mg} / \mathrm{kg}$ significantly inhibits hyperglycemia-induced the up-regulation of NF-KB protein levels in the kidney [10]. Consistent with previous findings $[9,10]$, our study indicated that HG-induced the up-regulation of nuclear NF-KB/p65 was counteracted by EA $(30 \mu \mathrm{M})$ treatment. In our mouse model of STZ-induced DN, a significant increase in nuclear NF-KB/p65 was also observed in 
the kidney, while EA (100 mg/kg) treatment dramatically inhibited nuclear NF-KB/p65 and its downstream targets.

Up-regulation of miR-150-3p has been reported in the serum and urinary exosomal of patients with DN $[33,34]$, suggesting that miR-150-3p may contribute to the pathogenesis of DN and serve as a therapeutic target. In vitro cell experiments, our results found that miR-150-3p was up-regulated in podocytes and RTECs in response to HG stimulation. Moreover, knockdown of miR-150-3p prevented HG-induced podocyte and RTEC apoptosis. Furthermore, the elevation of miR-150-3p was also exhibited in the kidney

of diabetic mice. To determine whether inhibition of miR-150-3p protected against hyperglycemia-induced renal dysfunction, miR-150-3p antagomir was applied in STZ-treated mice. After anti-miR-150-3p treatment for 8 weeks, renal function, tubular injury and interstitial fibrosis were obviously improved in diabetic mice. These findings provide a theoretical basis to treat DN by inhibiting miR-150-3p.

The previous study exhibits that over-activation of NF-KB accelerates miR-150-3p expression to inhibit osteogenesis [26]. In addition, cyclophosphamide induces lung injury in rats by promoting NF-KBmediated up-regulation of miR-150-3p [25]. These findings indicate that the NF-KB/miR-150-3p signaling axis may be associated with tissue injury. Intriguingly, both NF-KB and miR-150-3p were increased in HGstimulated podocytes and RTECs and kidneys of STZ-treated mice. EA administration neutralized HG- or hyperglycemia-induced the up-regulation of NF-KB and miR-150-3p in vitro and in vivo experimental models. Our finding indicated that the NF-KB/miR-150-3p signaling axis might be a potential target of EA to prevent renal injuries.

BCL2 is an apoptosis-related protein, and the disruption of the expression of BCL2 influences organic lesions [35]. miR-mediated post-transcriptional repression of BCL2 contributes to podocyte apoptosis in diabetic circumstances [36]. Our study revealed that BCL2 is a direct target of miR-150-3p, and overexpression of miR-150-3p significantly repressed BCL2 protein expression. Inhibition of miR-150-3p reversed hyperglycemia-induced the down-regulation of BCL2 protein. Moreover, silencing of miR-150-3p attenuated HG-induced podocyte and RTEC apoptosis, suggesting that interception of miR-150-3pmediated BCL2 repression may be a novel approach to improve renal injuries.

In conclusion, our findings revealed that EA modulated the suppression of NF-KB/miR-150-3p to activate $\mathrm{BCL} 2$ that contributed to prevent hyperglycemia-induced renal dysfunction. In addition, synthetic miR150-3p antagomir or inhibitors could alleviate tubular injury and interstitial fibrosis, and prevent HGinduced podocyte and RTEC apoptosis. These findings showed that miR-150-3p antagomir might provide a prospective therapeutic strategy for the treatment of DN.

\section{Declarations}

Ethics approval: This research was approved by the Ethics Committee of the Lihuili Hospital.

Consent for publication: Not applicable. 
Availability of data and material: The data that support the findings of this study are available from the corresponding author upon reasonable request.

Funding: Not applicable.

Competing interests: The authors declare that they have no competing interests.

Authors' contributions: Study design: Y-C; Literature research, Data acquisition and Data analysis: Y-C, Y$\mathrm{X}, \mathrm{Q}-\mathrm{N}, \mathrm{B}-\mathrm{G}, \mathrm{S}-\mathrm{C}, \mathrm{X}-\mathrm{J}$ and Q-W; Manuscript preparation and Manuscript editing: $\mathrm{Y}-\mathrm{C}$ and $\mathrm{Y}-\mathrm{X}$; Manuscript review: Y-C; Cell and animal experiments: Y-C, Y-X, Q-N, B-G, S-C, X-J and Q-W.

All authors read and approved the final manuscript.

\section{References}

1. Huang, F., Q. Wang, F. Guo, Y. Zhao, L. Ji, T. An, Y. Song, Y. Liu, Y. He, and G. Qin. 2019. FoxO1mediated inhibition of STAT1 alleviates tubulointerstitial fibrosis and tubule apoptosis in diabetic kidney disease. EBioMedicine 48: 491-504.

2. Kato, T., M. Hagiyama, Y. Takashima, A. Yoneshige, and A. Ito. 2018. Cell adhesion molecule-1 shedding induces apoptosis of renal epithelial cells and exacerbates human nephropathies. Am J Physiol Renal Physio/314: F388-Ff398.

3. Brezniceanu, M. L., C. J. Lau, N. Godin, I. Chénier, A. Duclos, J. Ethier, J. G. Filep, J. R. Ingelfinger, S. L. Zhang, and J. S. Chan. 2010. Reactive oxygen species promote caspase-12 expression and tubular apoptosis in diabetic nephropathy. J Am Soc Nephrol 21: 943-954.

4. Alfei, S., F. Turrini, S. Catena, P. Zunin, M. Grilli, A. M. Pittaluga, and R. Boggia. 2019. Ellagic acid a multi-target bioactive compound for drug discovery in CNS? A narrative review. Eur J Med Chem 183: 111724.

5. Ríos, J. L., R. M. Giner, M. Marín, and M. C. Recio. 2018. A Pharmacological Update of Ellagic Acid. Planta Med 84: 1068-1093.

6. Ahmed, T., W. N. Setzer, S. F. Nabavi, I. E. Orhan, N. Braidy, E. Sobarzo-Sanchez, and S. M. Nabavi. 2016. Insights Into Effects of Ellagic Acid on the Nervous System: A Mini Review. Curr Pharm Des 22: 1350-1360.

7. Kannan, M. M., and S. D. Quine. 2013. Ellagic acid inhibits cardiac arrhythmias, hypertrophy and hyperlipidaemia during myocardial infarction in rats. Metabolism 62: 52-61.

8. Wang, L., Y. Wei, C. Ning, M. Zhang, P. Fan, D. Lei, J. Du, M. Gale, Y. Ma, and Y. Yang. 2019. Ellagic acid promotes browning of white adipose tissues in high-fat diet-induced obesity in rats through suppressing white adipocyte maintaining genes. Endocr J 66: 923-936.

9. Ahad, A., A. A. Ganai, M. Mujeeb, and W. A. Siddiqui. 2014. Ellagic acid, an NF-kB inhibitor, ameliorates renal function in experimental diabetic nephropathy. Chem Biol Interact 219: 64-75.

10. Zhou, B., Q. Li, J. Wang, P. Chen, and S. Jiang. 2019. Ellagic acid attenuates streptozocin induced diabetic nephropathy via the regulation of oxidative stress and inflammatory signaling. Food Chem 
Toxicol123: 16-27.

11. Raghu, G., S. Jakhotia, P. Yadagiri Reddy, P. A. Kumar, and G. Bhanuprakash Reddy. 2016. Ellagic acid inhibits non-enzymatic glycation and prevents proteinuria in diabetic rats. Food Funct 7: 1574-1583.

12. Ding, X., T. Jian, Y. Wu, Y. Zuo, J. Li, H. Lv, and L. Ma, et al. 2019. Ellagic acid ameliorates oxidative stress and insulin resistance in high glucose-treated HepG2 cells via miR-223/keap1-Nrf2 pathway. Biomed Pharmacother110: 85-94.

13. Navickas, R., D. Gal, A. Laucevičius, A. Taparauskaitè, M. Zdanytè, and P. Holvoet. 2016. Identifying circulating microRNAs as biomarkers of cardiovascular disease: a systematic review. Cardiovasc Res 111: 322-337.

14. Yaribeygi, H., N. Katsiki, B. Behnam, H. Iranpanah, and A. Sahebkar. 2018. MicroRNAs and type 2 diabetes mellitus: Molecular mechanisms and the effect of antidiabetic drug treatment. Metabolism 87: 48-55.

15. Tao, H., Z. Y. Song, X. S. Ding, J. J. Yang, K. H. Shi, and J. Li. 2018. LncRNAs and miRs as epigenetic signatures in diabetic cardiac fibrosis: new advances and perspectives. Endocrine 62: 281-291.

16. Sankrityayan, H., Y. A. Kulkarni, and A. B. Gaikwad. 2019. Diabetic nephropathy: The regulatory interplay between epigenetics and microRNAs. Pharmacol Res 141: 574-585.

17. He, M., J. Wang, Z. Yin, Y. Zhao, H. Hou, J. Fan, and H. Li, et al. 2019. MiR-320a induces diabetic nephropathy via inhibiting MafB. Aging (Albany NY) 11: 3055-3079.

18. Sun, Z., Y. Ma, F. Chen, S. Wang, B. Chen, and J. Shi. 2018. miR-133b and miR-199b knockdown attenuate TGF- $\beta 1$-induced epithelial to mesenchymal transition and renal fibrosis by targeting SIRT1 in diabetic nephropathy. Eur J Pharmaco/837: 96-104.

19. Tsai, Y. C., M. C. Kuo, W. W. Hung, L. Y. Wu, P. H. Wu, W. A. Chang, P. L. Kuo, and Y. L. Hsu. 2020. High Glucose Induces Mesangial Cell Apoptosis through miR-15b-5p and Promotes Diabetic Nephropathy by Extracellular Vesicle Delivery. Mol Ther 28: 963-974.

20. Wei, D. Z., C. Lin, Y. Q. Huang, L. P. Wu, and M. Y. Huang. 2017. Ellagic acid promotes ventricular remodeling after acute myocardial infarction by up-regulating miR-140-3p. Biomed Pharmacother 95 : 983-989.

21. Percie du Sert, N., and A. Ahluwalia. 2020. Reporting animal research: Explanation and elaboration for the ARRIVE guidelines 2.0. PLoS Biol 18: e3000411.

22. Yu, X., X. Meng, M. Xu, X. Zhang, Y. Zhang, G. Ding, S. Huang, A. Zhang, and Z. Jia. 2018. Celastrol ameliorates cisplatin nephrotoxicity by inhibiting NF-KB and improving mitochondrial function. EBioMedicine 36: 266-280.

23. Zhang, Y., J. Kong, D. K. Deb, A. Chang, and Y. C. Li. 2010. Vitamin D receptor attenuates renal fibrosis by suppressing the renin-angiotensin system. J Am Soc Nephrol 21: 966-973.

24. Han, P., W. Weng, Y. Chen, Y. Cai, Y. Wang, M. Wang, and H. Zhan, et al. 2020. Niclosamide ethanolamine attenuates systemic lupus erythematosus and lupus nephritis in MRL/lpr mice. Am J Transl Res 12: 5015-5031. 
25. Abdel-Latif, G. A., A. H. A. Elwahab, R. A. Hasan, N. F. ElMongy, and M. M. Ramzy. 2020. A novel protective role of sacubitril/valsartan in cyclophosphamide induced lung injury in rats: impact of miRNA-150-3p on NF-KB/MAPK signaling trajectories. Sci Rep 10: 13045.

26. Wang, N., Z. Zhou, T. Wu, W. Liu, P. Yin, and C. Pan, and X. Yu. 2016. TNF-a-induced NF-kB activation upregulates microRNA-150-3p and inhibits osteogenesis of mesenchymal stem cells by targeting $\beta$ catenin. Open Biol 6.

27. White, S., and L. Lin. 2020. NF-KB and tPA Signaling in Kidney and Other Diseases. Cells 9.

28. Tang, S. C. W., and W. H. Yiu. 2020. Innate immunity in diabetic kidney disease. Nat Rev Nephro/ 16: 206-222.

29. Wada, J., and H. Makino. 2016. Innate immunity in diabetes and diabetic nephropathy. Nat Rev Nephrol 12: 13-26.

30. Niu, X., Q. Yao, W. Li, L. Zang, W. Li, J. Zhao, F. Liu, and W. Zhi. 2019. Harmine mitigates LPS-induced acute kidney injury through inhibition of the TLR4-NF-KB/NLRP3 inflammasome signalling pathway in mice. Eur J Pharmacol 849: 160-169.

31. Chen, Y., J. Qiu, B. Chen, Y. Lin, Y. Chen, G. Xie, J. Qiu, H. Tong, and D. Jiang. 2018. Long non-coding RNA NEAT1 plays an important role in sepsis-induced acute kidney injury by targeting miR-204 and modulating the NF-KB pathway. Int Immunopharmacol 59: 252-260.

32. Song, W., L. Wei, Y. Du, Y. Wang, and S. Jiang. 2018. Protective effect of ginsenoside metabolite compound $\mathrm{K}$ against diabetic nephropathy by inhibiting NLRP3 inflammasome activation and NF$\mathrm{KB} / \mathrm{p} 38$ signaling pathway in high-fat diet/streptozotocin-induced diabetic mice. Int Immunopharmacol63: 227-238.

33. Kim, H., Y. U. Bae, J. S. Jeon, H. Noh, H. K. Park, D. W. Byun, D. C. Han, S. Ryu, and S. H. Kwon. 2019. The circulating exosomal microRNAs related to albuminuria in patients with diabetic nephropathy. $J$ Transl Med 17: 236.

34. Lee, W. C., L. C. Li, H. Y. Ng, and P. T. Lin. 2020. Urinary Exosomal MicroRNA Signatures in Nephrotic, Biopsy-Proven Diabetic Nephropathy. J Clin Med 9.

35. Yamaguchi, M. 2013. The anti-apoptotic effect of regucalcin is mediated through multisignaling pathways. Apoptosis 18: 1145-1153.

36. Chen, Y. Q., X. X. Wang, X. M. Yao, D. L. Zhang, X. F. Yang, S. F. Tian, and N. S. Wang. 2011. MicroRNA-195 promotes apoptosis in mouse podocytes via enhanced caspase activity driven by BCL2 insufficiency. Am J Nephro/34: 549-559.

\section{Figures}




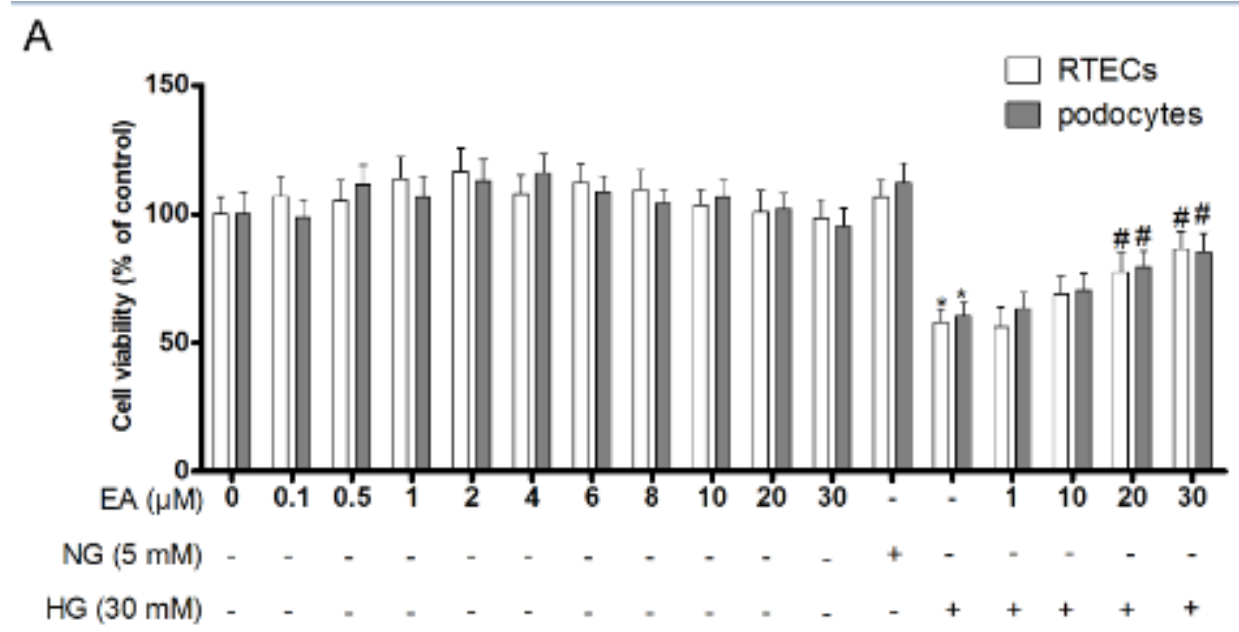

B

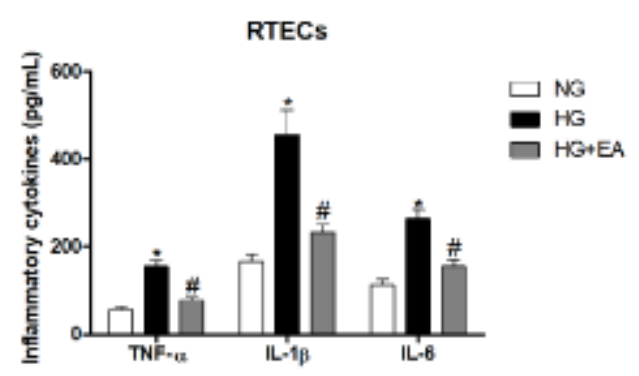

C
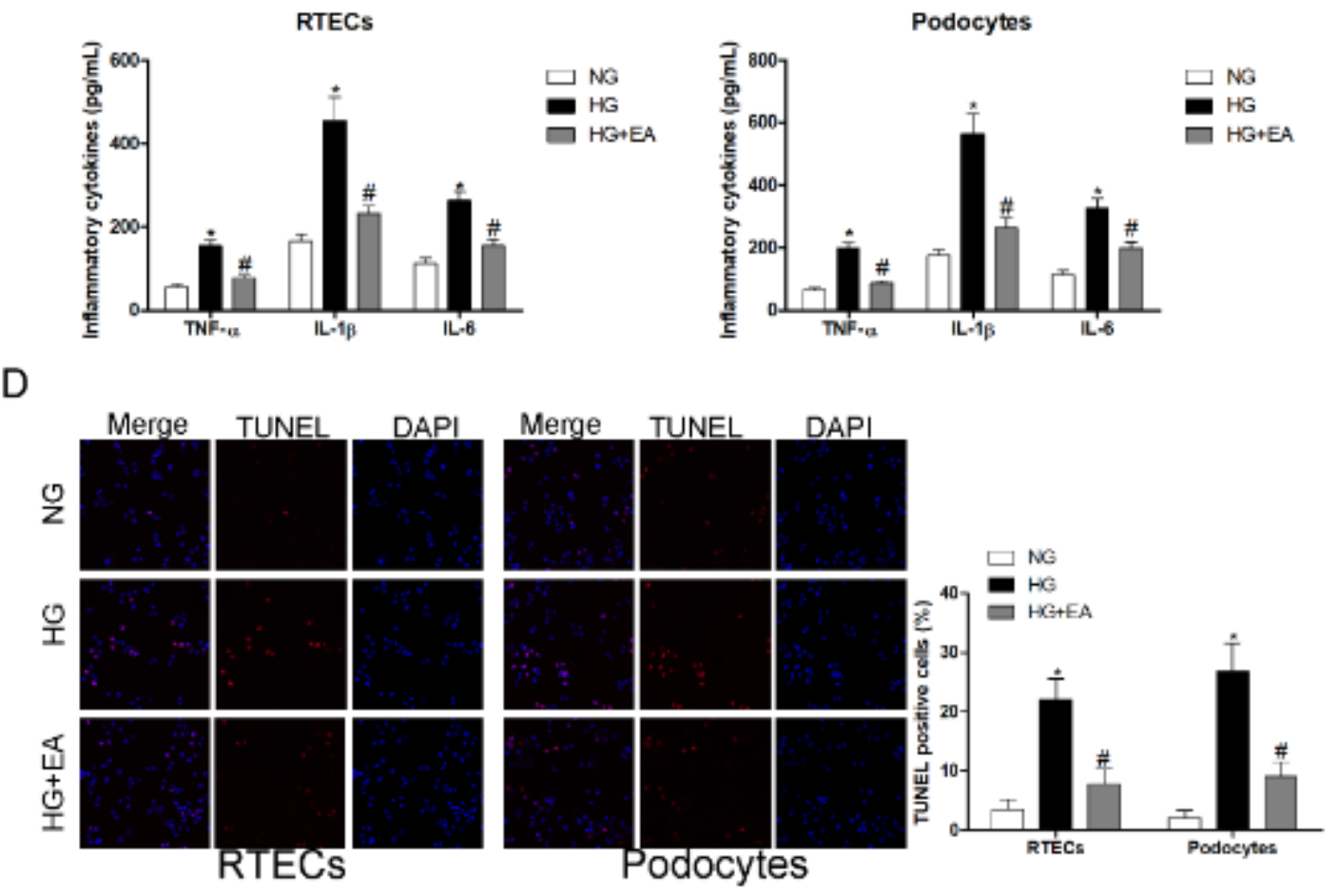

Figure 1

EA attenuates inflammatory response apoptosis in HG-stimulated RTECs and podocytes. The effect of $\mathrm{NG}, \mathrm{HG}$ and EA on podocyte and RTEC growth in vitro was evaluated by CCK8 assays after $24 \mathrm{~h}$ treatment (A). Proinflammatory cytokines, TNF-a, IL-1 $\beta$ and IL-6, were detected by ELISA assays in HG-stimulated podocytes (B) and RTECs (C) with or without EA treatment for $24 \mathrm{~h}$. After HG-stimulated podocytes and RTECs with or without EA treatment for $24 \mathrm{~h}$, TUNEL positive staining cells were analyzed using TUNEL staining (D). ${ }^{*} P<0.05$ vs NG group; \# $P<0.05$ vs HG group. $n=3$ in each group. 
A

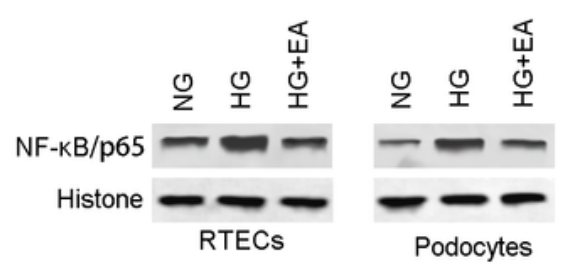

C

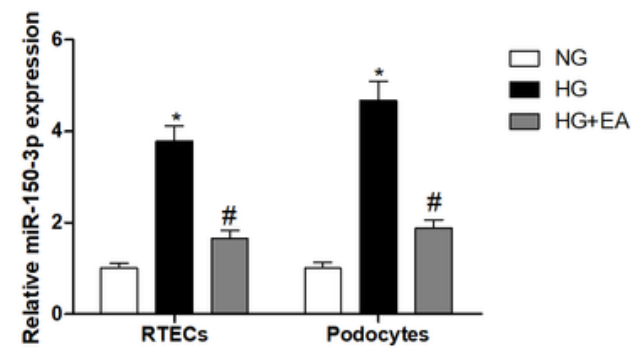

B

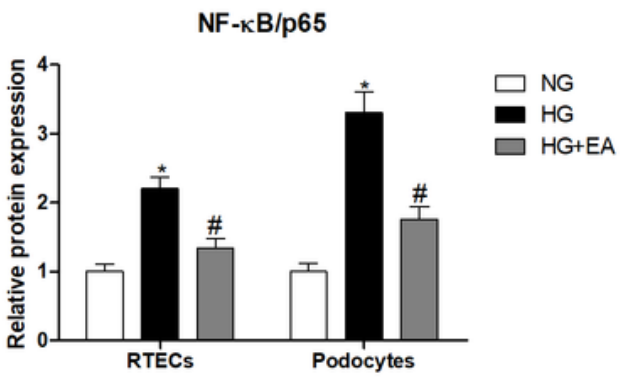

D

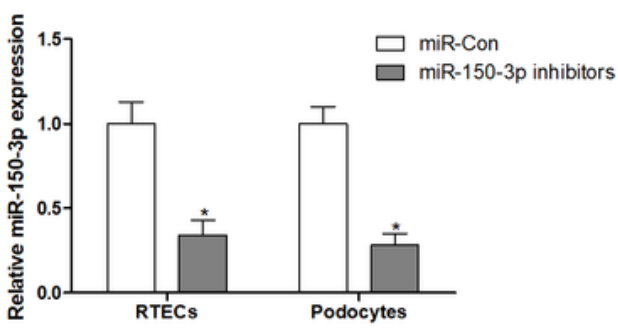

E

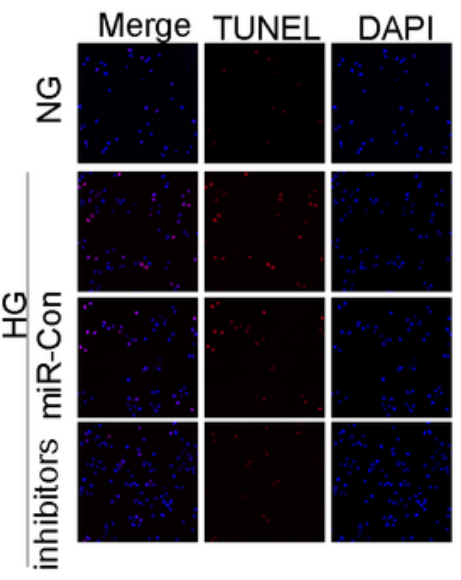

$\mathrm{F}$

RTEC

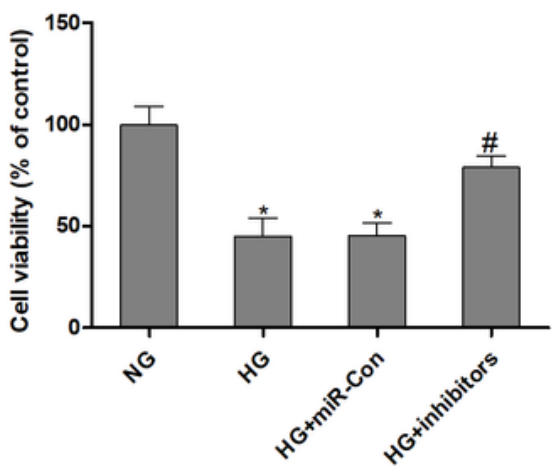

Merge TUNEL DAP
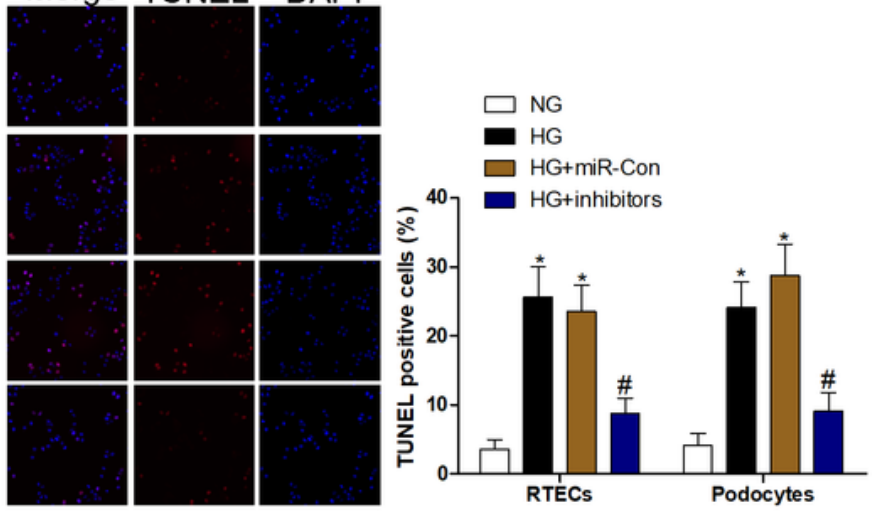

Podocytes

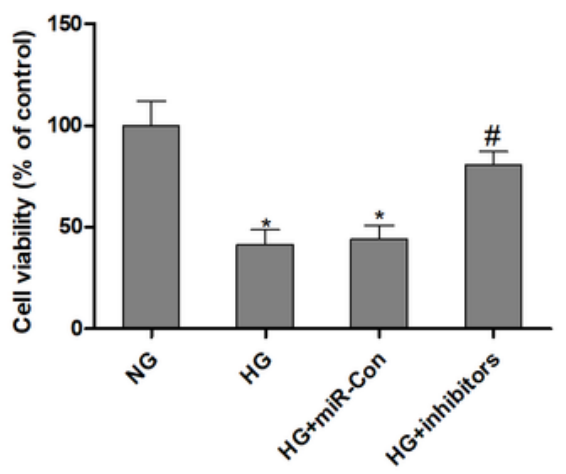

Figure 2

EA mediates the NF-KB/miR-150-3p axis in HG-induced RTEC and podocyte apoptosis. After HGstimulated podocytes and RTECs with or without EA treatment for $24 \mathrm{~h}, \mathrm{NF}-\mathrm{kB} / \mathrm{p} 65$ protein expression in the nucleus was measured using western blot ( $A$ and $B$ ); miR-150-3p expression was detected by RT-qPCR (C). After transfection with miR-Con or miR-150-3p inhibitors into podocytes and RTECs for $24 \mathrm{~h}$, miR-150$3 p$ expression was detected by RT-qPCR (D). After transfection with miR-Con or miR-150-3p inhibitors into 
HG-exposed podocytes and RTECs for $24 \mathrm{~h}$, apoptosis rate and cell viability were evaluated by TUNEL staining $(E)$ and CCK8 assays $(F)$, respectively. * $P<0.05$ vs NG group; \# $P<0.05$ vs HG group. $n=3$ in each group.

A

mmu-miR-150-3p 3'-GAUAGGGGGUCCGGACAUGGUC-5'

ll||l||

BCL2 5'-AUGCAUUAUCAAUGAUGUACCAU-3'

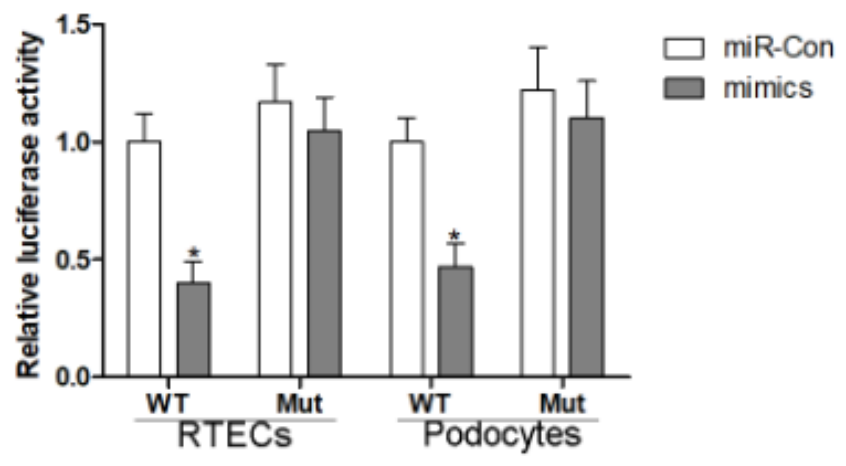

C

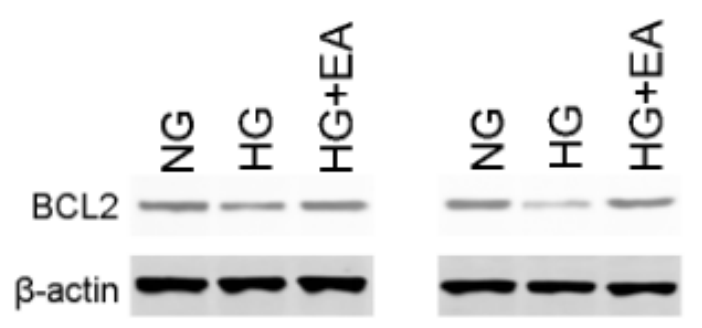

B
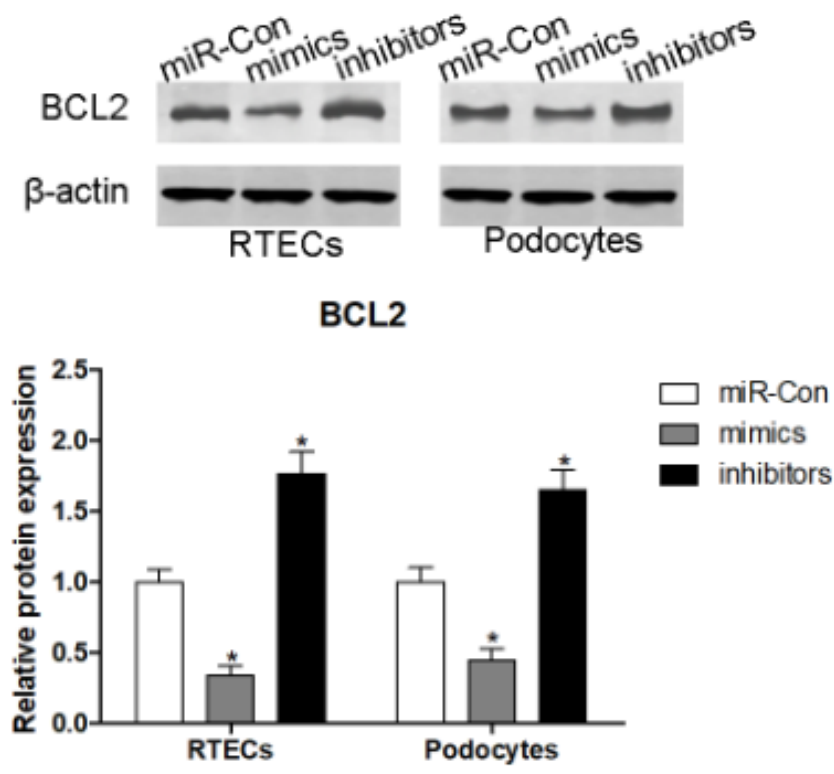

BCL2

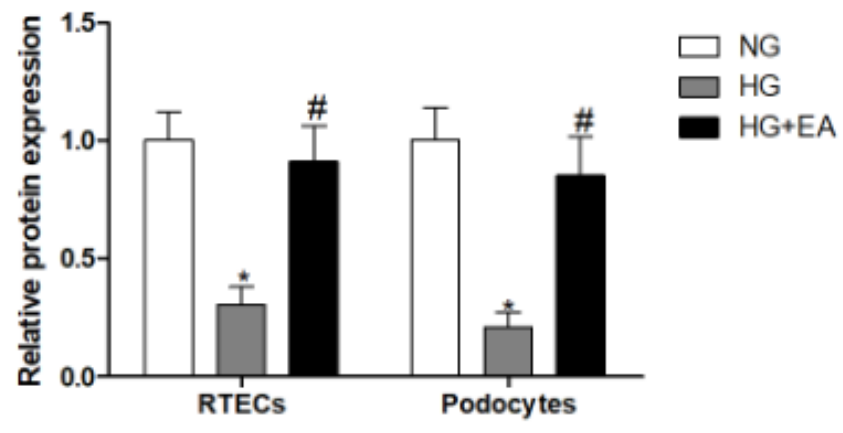

Figure 3

BCL2 expression can be regulated by miR-150-3p and EA. Binding sites between miR-150-3p and BCL2 were predicted by on-line bioinformatics algorithm, TargetScan; and luciferase assays were performed to investigate a direct association between miR-150-3p and BCL2 (A). After transfection with miR-Con, miR150-3p mimics or inhibitors into podocytes and RTECs for $24 \mathrm{~h}, \mathrm{BCL} 2$ protein expression was measured using western blot (B). After podocytes and RTECs exposure to HG with or without EA treatment for $24 \mathrm{~h}$,

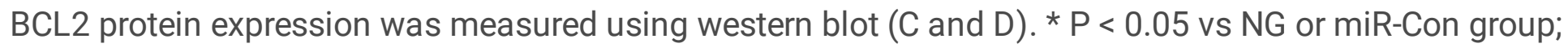
$\# P<0.05$ vs HG group. $n=3$ in each group. 


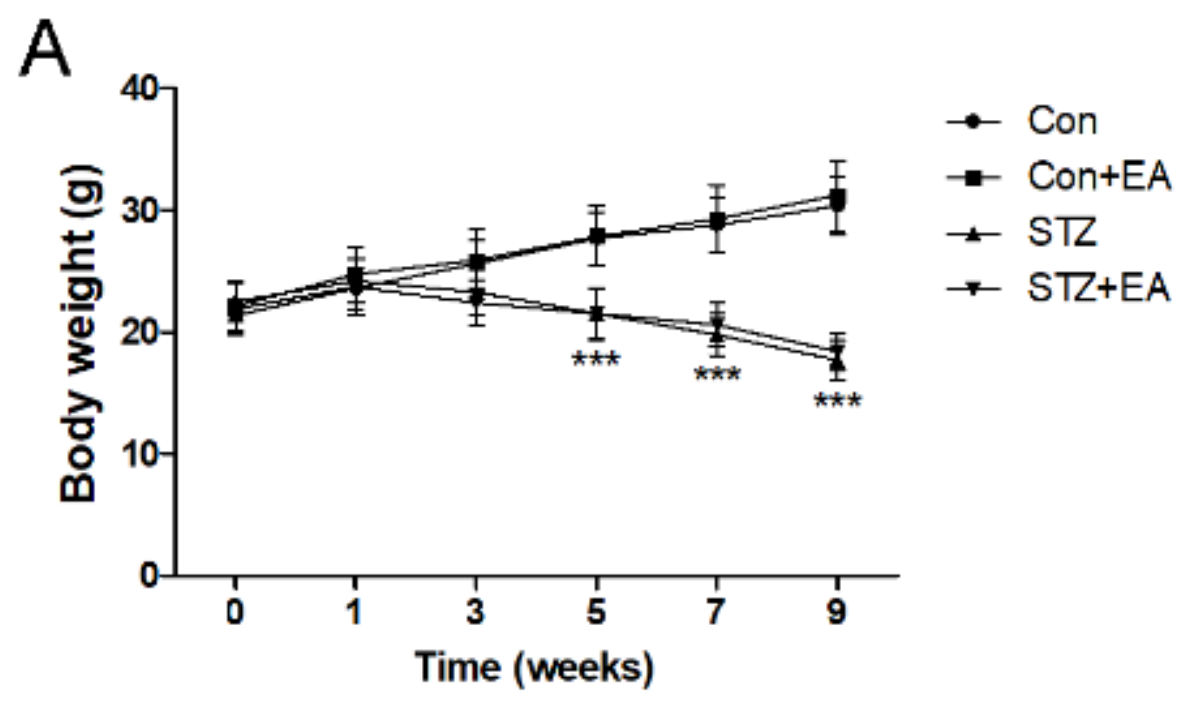

B

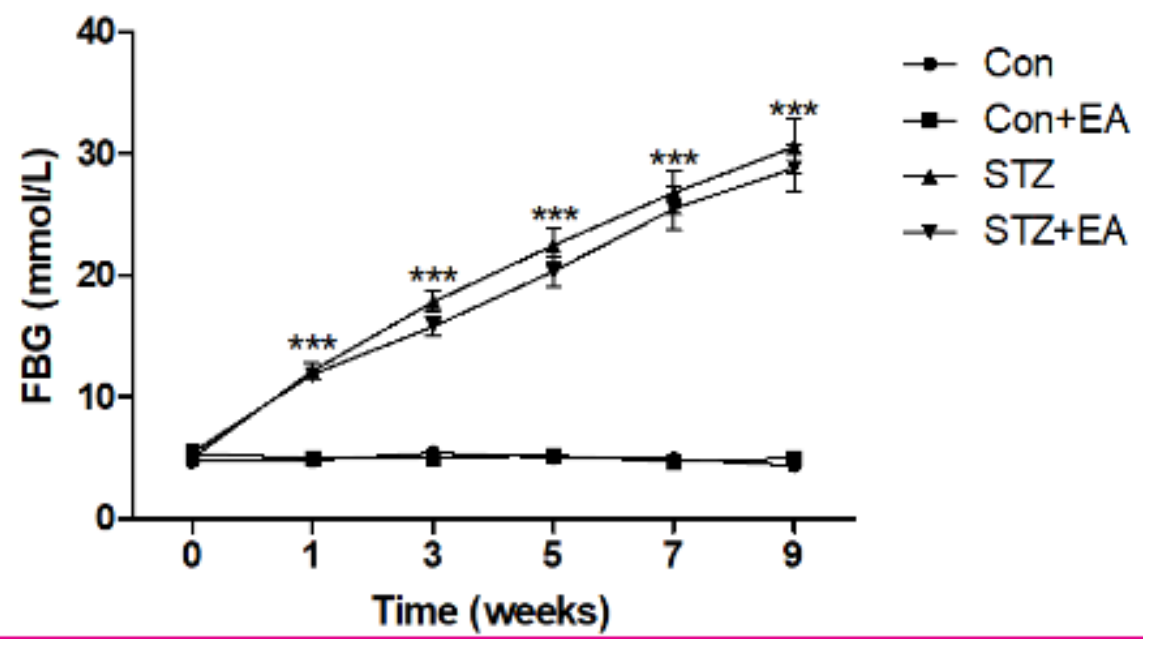

Figure 4

EA has no effect on body weight and FBG in diabetic mice. Body weight (A) and FBG (B) were recorded every two weeks after EA treatment in the experimental period for 8 weeks. ${ }^{*} * \mathrm{P}<0.001$ represents STZ or STZ+EA treatment group vs Con group. 
A

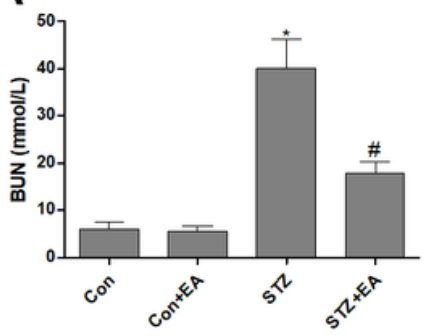

C

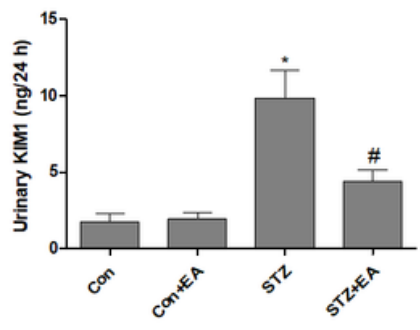

F

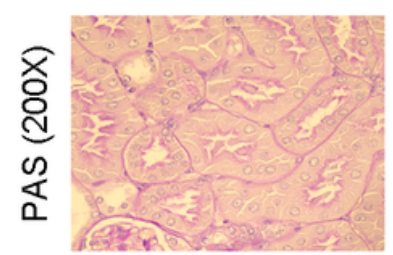

G
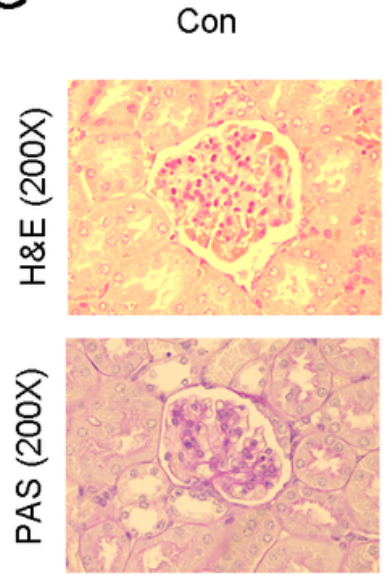
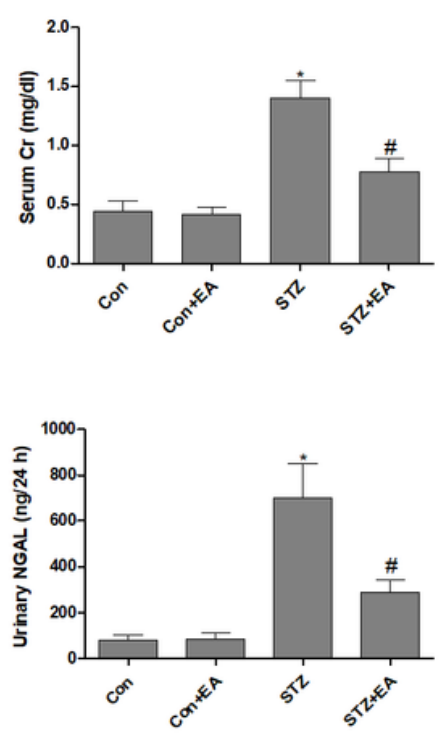

Con+EA

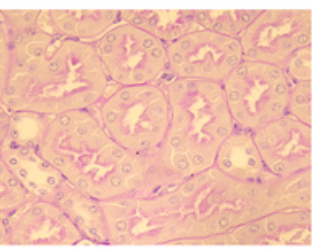

Con+EA
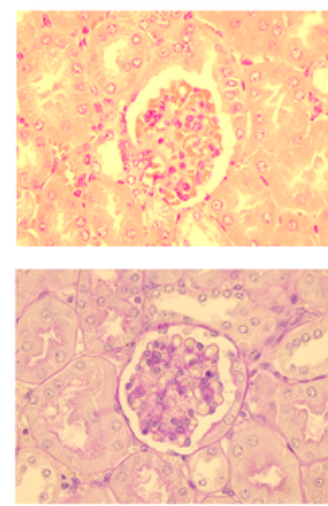

B

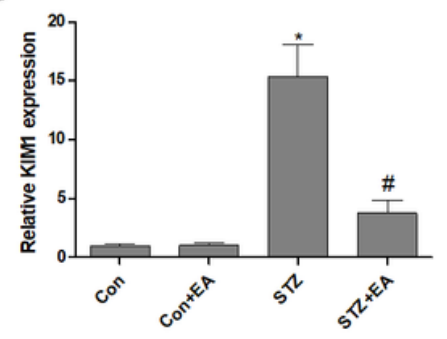

D

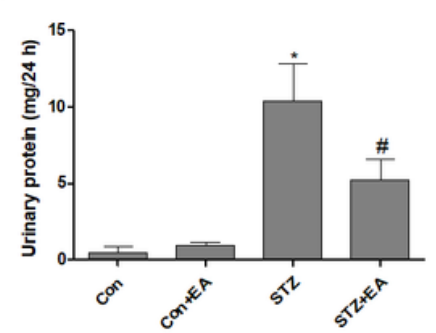

STZ

$S T Z+E A$
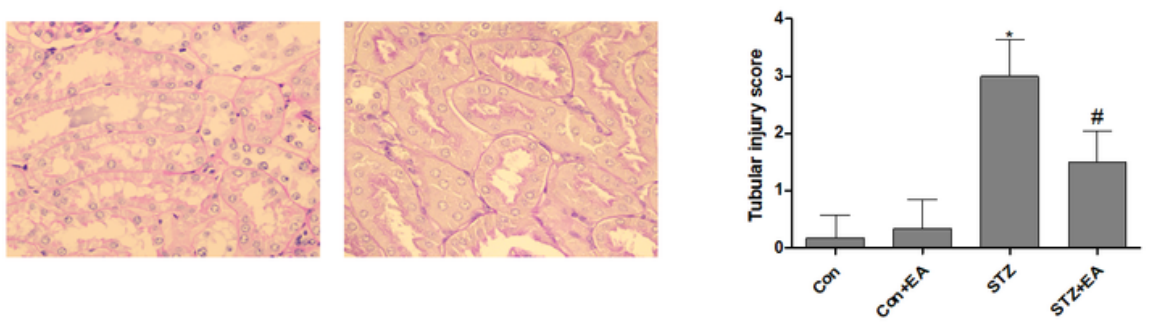

STZ
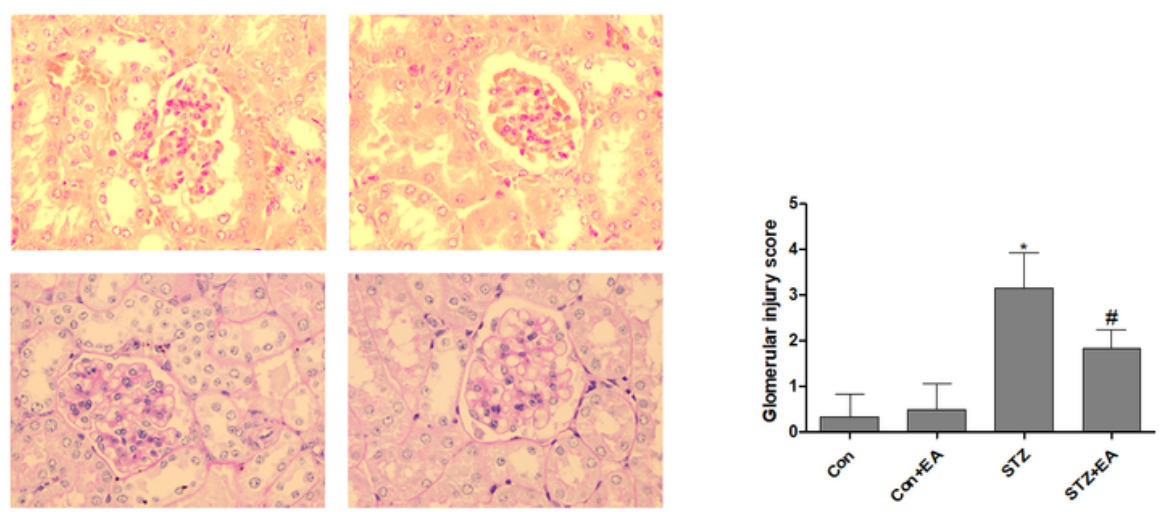

Figure 5

EA mitigates renal injury in diabetic mice. After normal or diabetic mice were administrated by EA (100 $\mathrm{mg} / \mathrm{kg} /$ day) for 8 weeks, BUN and serum $\mathrm{Cr}$ (A), KIM1 and NGAL mRNA (B), urinary KIM1 and NGAL (C), urinary total protein (D) and renal nephrin mRNA expression (E) were used to evaluate renal injuries in diabetic mice. Tubular (F) and glomerular $(\mathrm{G})$ injury score were evaluated by PAS and H\&E staining. * $P<$ 0.05 vs Con group; \# $\mathrm{P}<0.05$ vs STZ group. $\mathrm{n}=6$ in each group. 
A

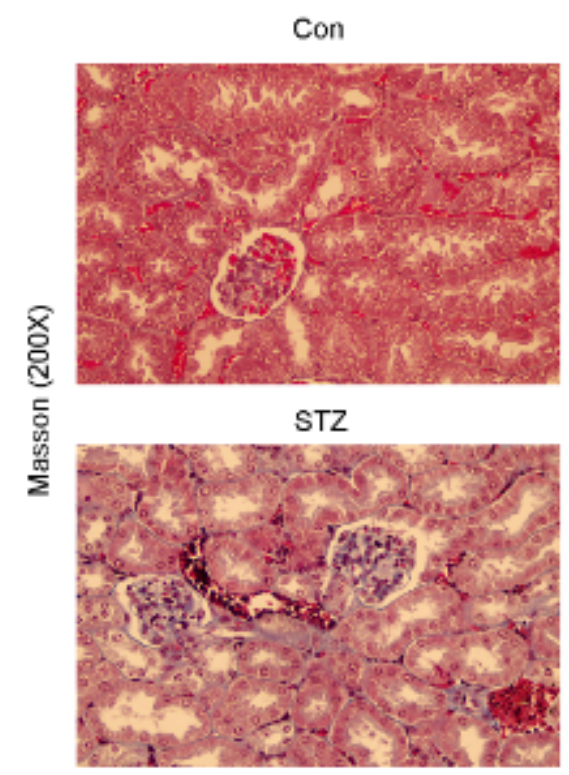

B

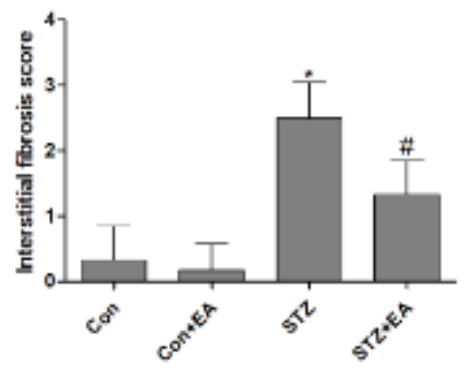

D

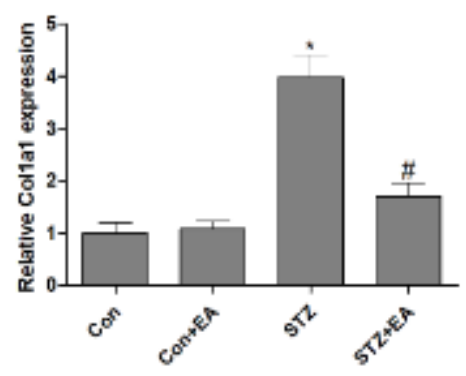

Con+EA

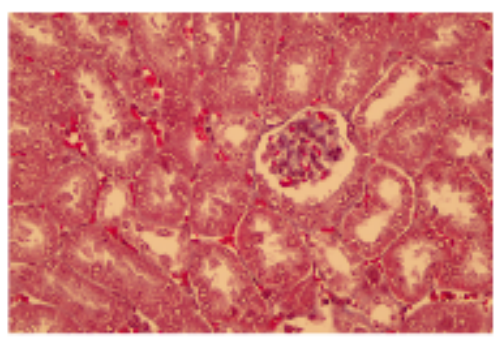

STZ+EA

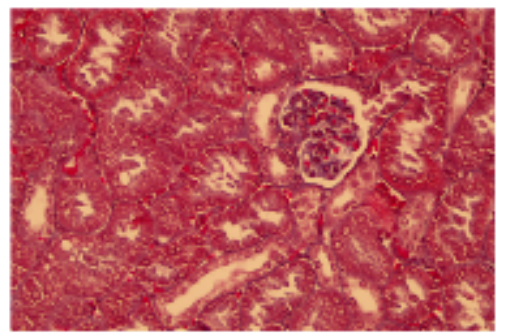

C

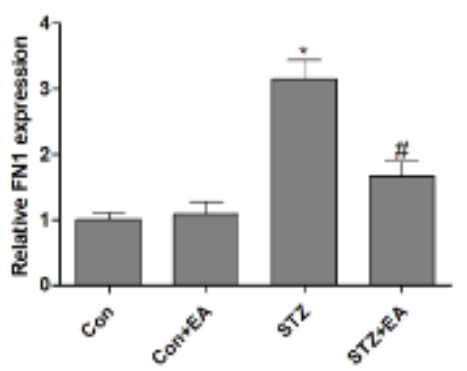

$\mathrm{E}$

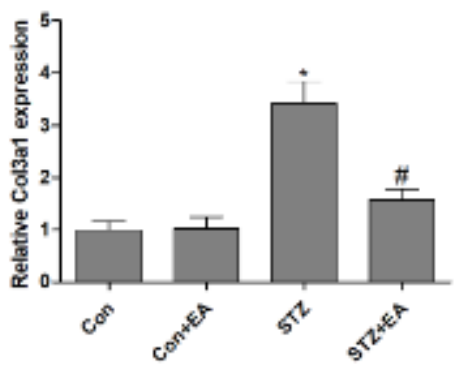

Figure 6

EA alleviates interstitial fibrosis in diabetic mice. After normal or diabetic mice was administrated by EA (100 mg/kg/day) for 8 weeks, renal interstitial fibrosis (A) and score (B) were evaluated by Masson's Trichrome staining. mRNA expression of FN1 (C), Col1a1 (D) and Col3a1 (E) was measured by RT-qPCR. * $P<0.05$ vs Con group; \# $P<0.05$ vs STZ group. $n=6$ in each group. 
A

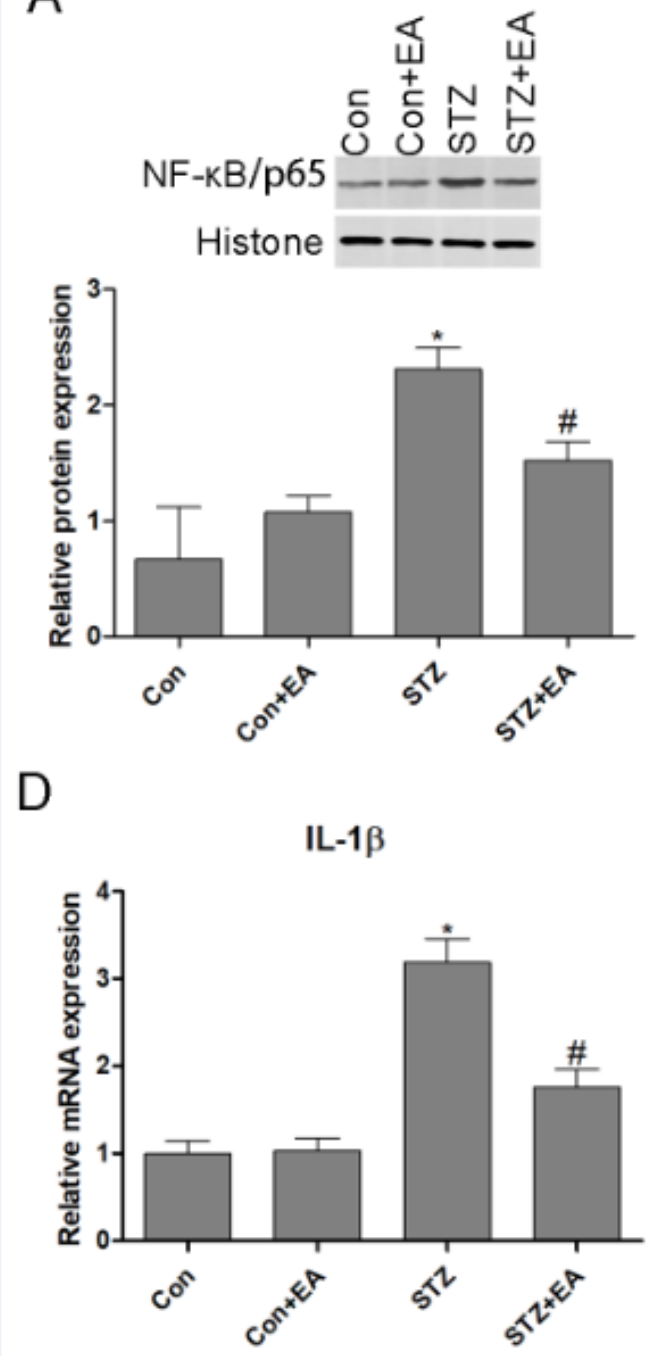

B

$E$

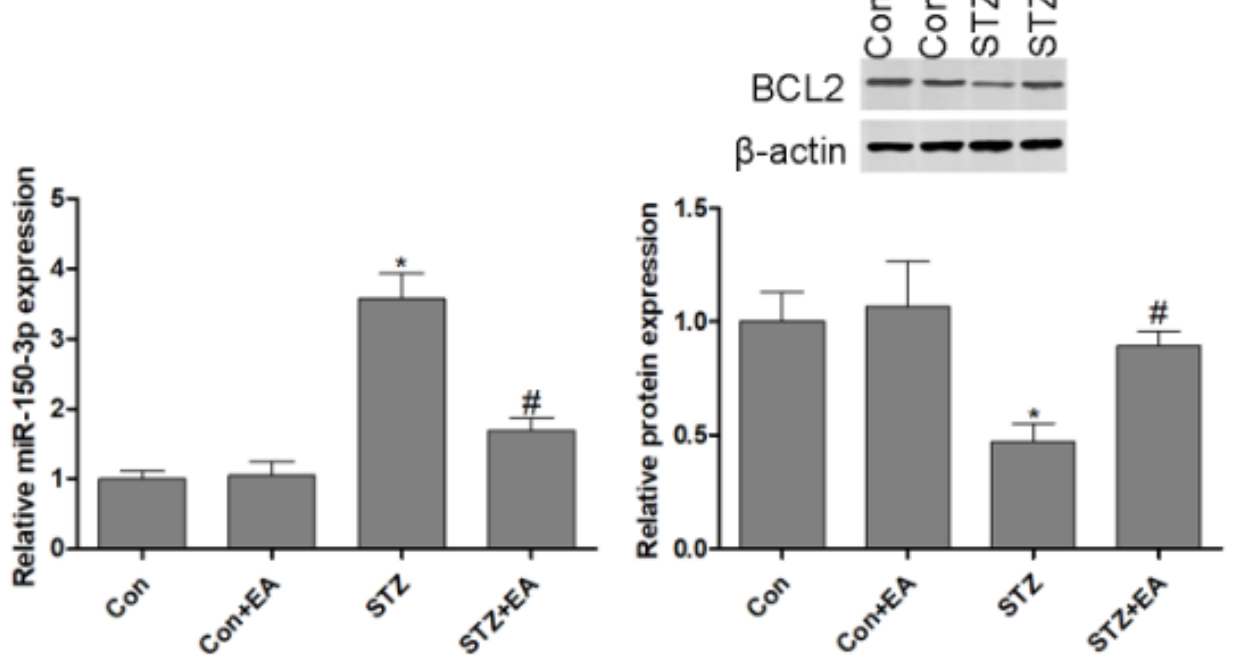

C

F

IL-6

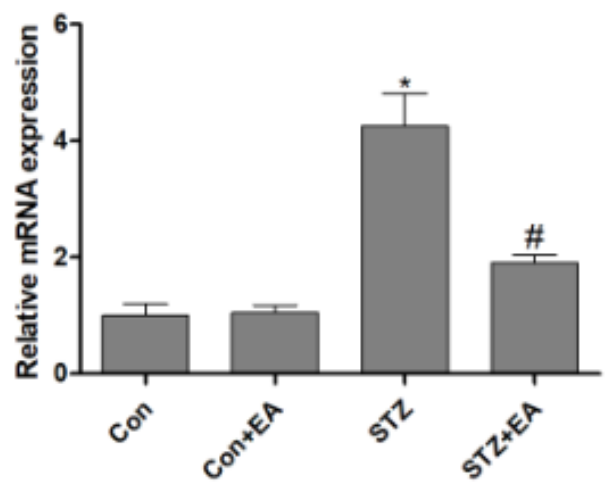

MCP1

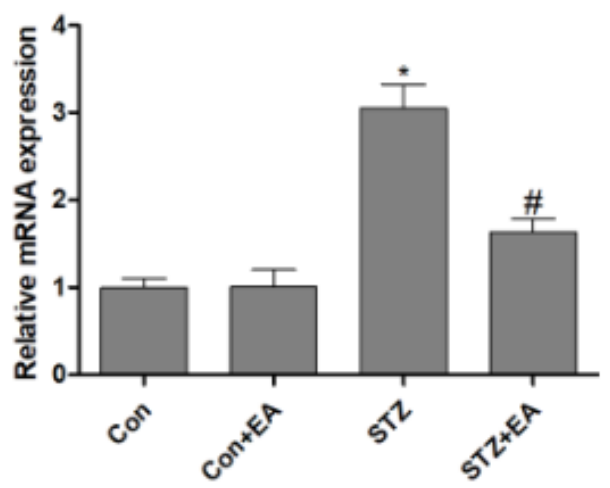

Figure 7

EA mediates NF-kB/miR-150-3p/BCL2 activity in diabetic mice. After normal or diabetic mice were administrated by EA (100 mg/kg/day) for 8 weeks, NF-KB/p65 protein (A), miR-150-3p (B) and BCL2 (C) protein levels were measured in the kidney. Pro-inflammatory cytokines and mediators, IL-1 $\beta$ (D), IL-6 (E) and MCP1 (F) mRNA expression in the kidney were measured using RT-qPCR. * $P<0.05$ vs Con group; \# P $<0.05$ vs STZ group. $\mathrm{n}=6$ in each group. 

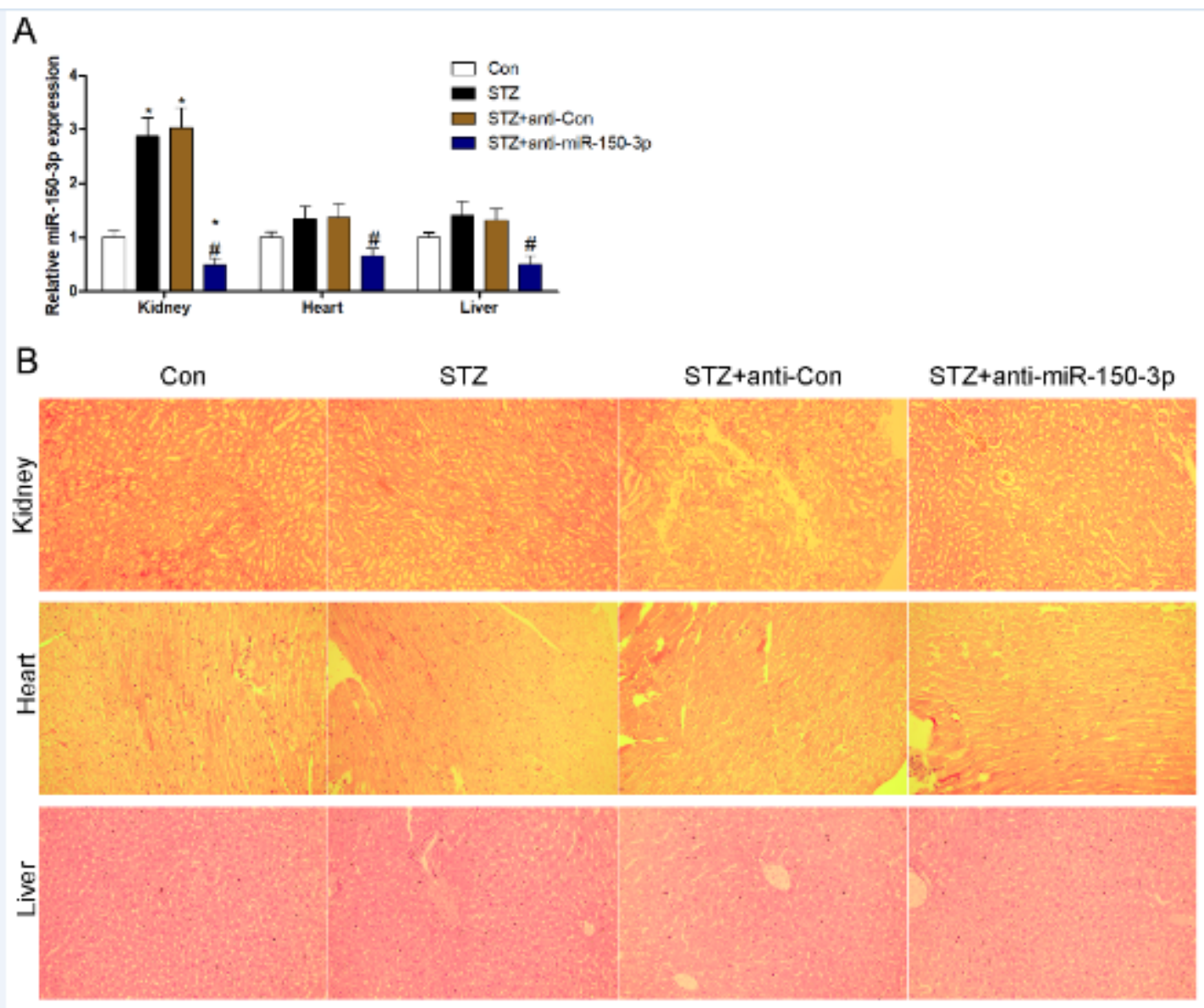

C
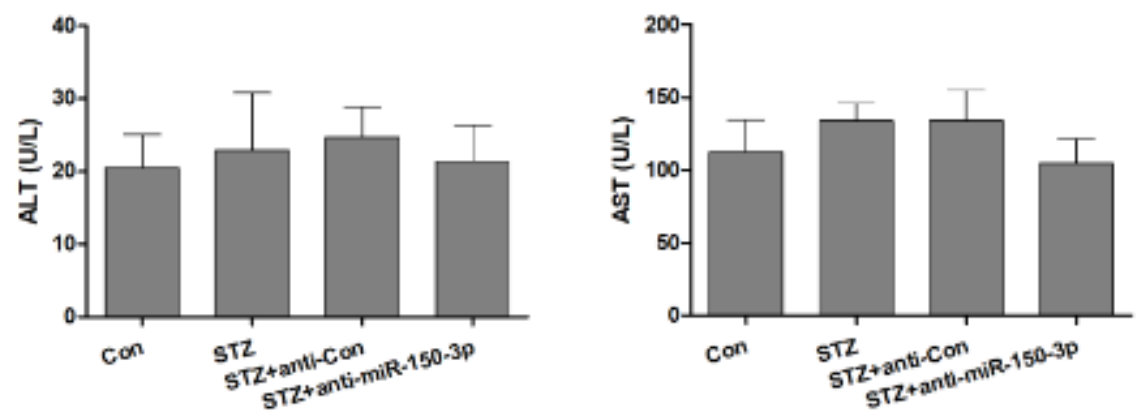

\section{Figure 8}

The effect of miR-150-3p antagomir in kidney, heart and liver of diabetic mice. After diabetic mice were treated with miR-150-3p antagomir (anti-miR-150-3p; $20 \mathrm{nM} / 0.1 \mathrm{~mL}$; once/two weeks) for 8 weeks, miR150-3p expression in kidney, heart and liver was measured using RT-qPCR (A). H\&E staining was used to evaluate histologic damage (B). Serum ALT and AST were measured using ELISA kits (C). * $P<0.05$ vs Con group; \# $\mathrm{P}<0.05$ vs STZ group. $\mathrm{n}=6$ in each group. 
A

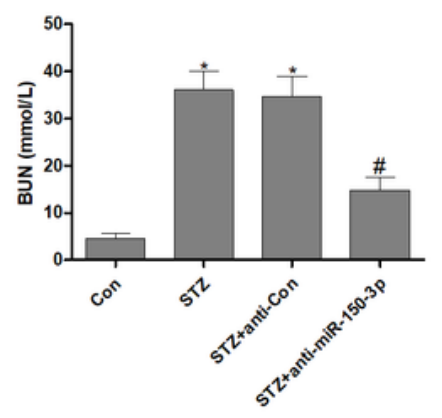

D Con STZ
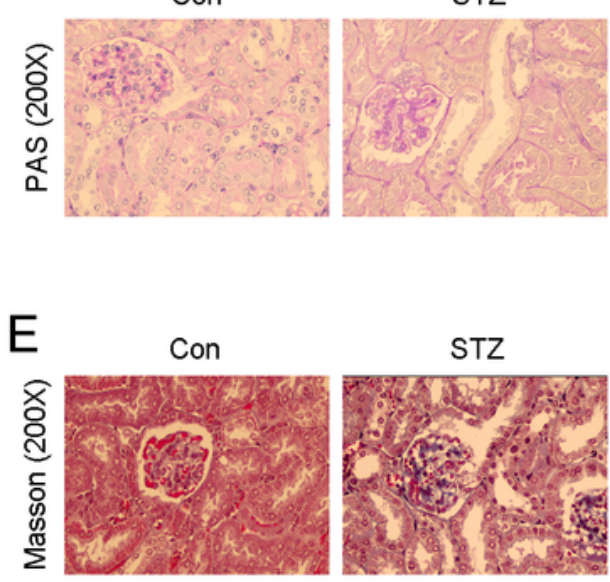

$\mathrm{F}$
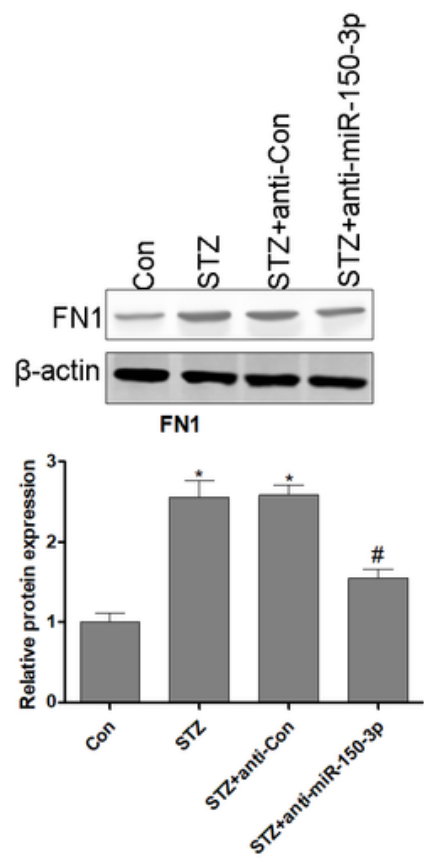

B

G

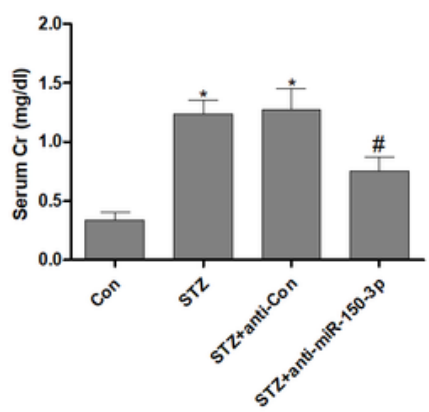

STZ+anti-Con
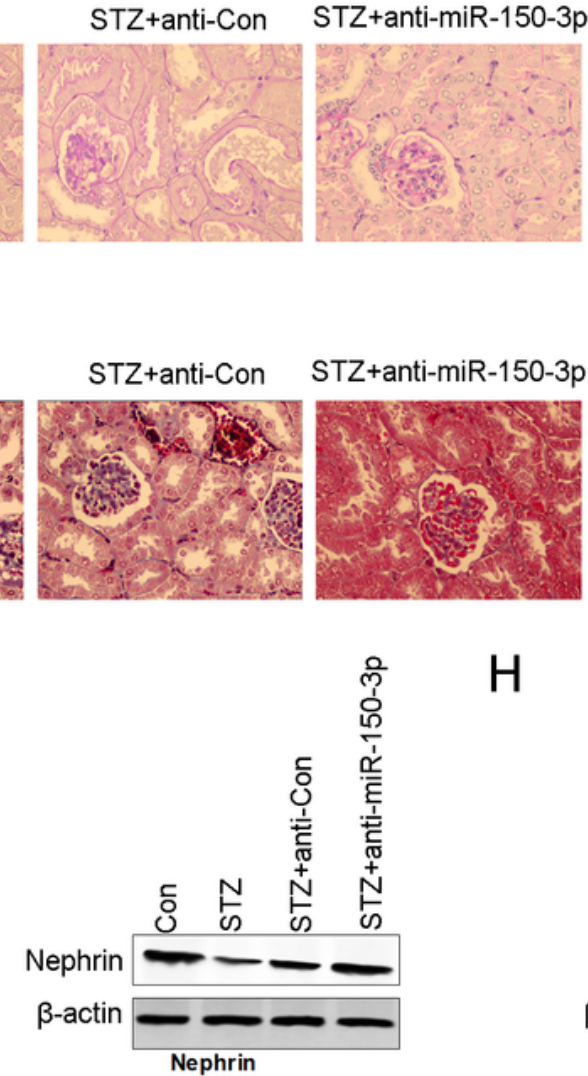

$\mathrm{H}$

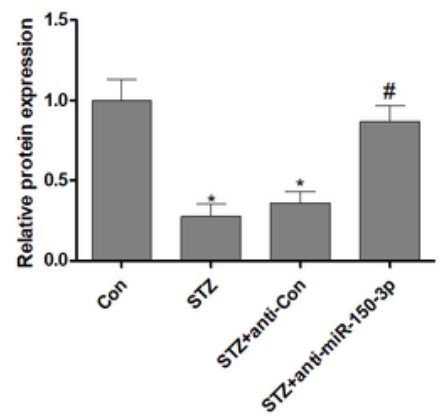

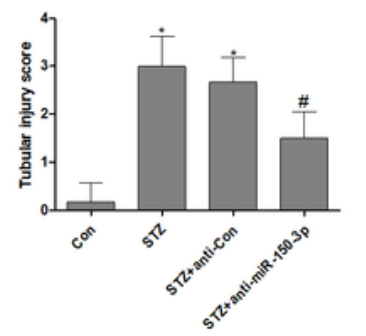
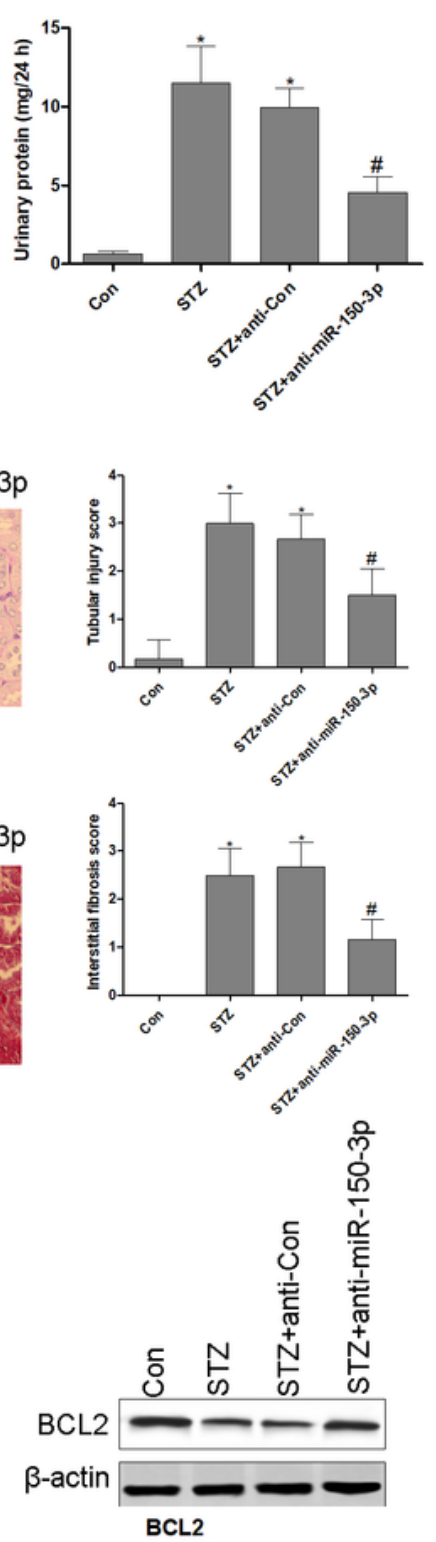

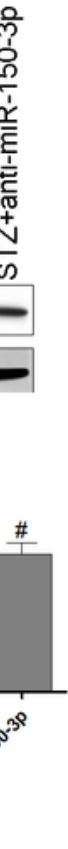

\section{Figure 9}

Anti-miR-150-3p treatment mitigates renal injury in diabetic mice. After diabetic mice were treated with miR-150-3p antagomir (anti-miR-150-3p; 20 nM/0.1 mL; once/two weeks) for 8 weeks, BUN (A), serum Cr (B) and urinary total protein (C) were used to evaluate renal injuries in diabetic mice. Renal tubular injury (D) and interstitial fibrosis (E) were evaluated using PAS and Masson's Trichrome staining, respectively. 
FN1 $(F)$, nephrin $(G)$ and BCL2 $(H)$ protein expression in the kidney were measured using western blot. * $P$ $<0.05$ vs Con group; \# $\mathrm{P}<0.05$ vs STZ group. $\mathrm{n}=6$ in each group.

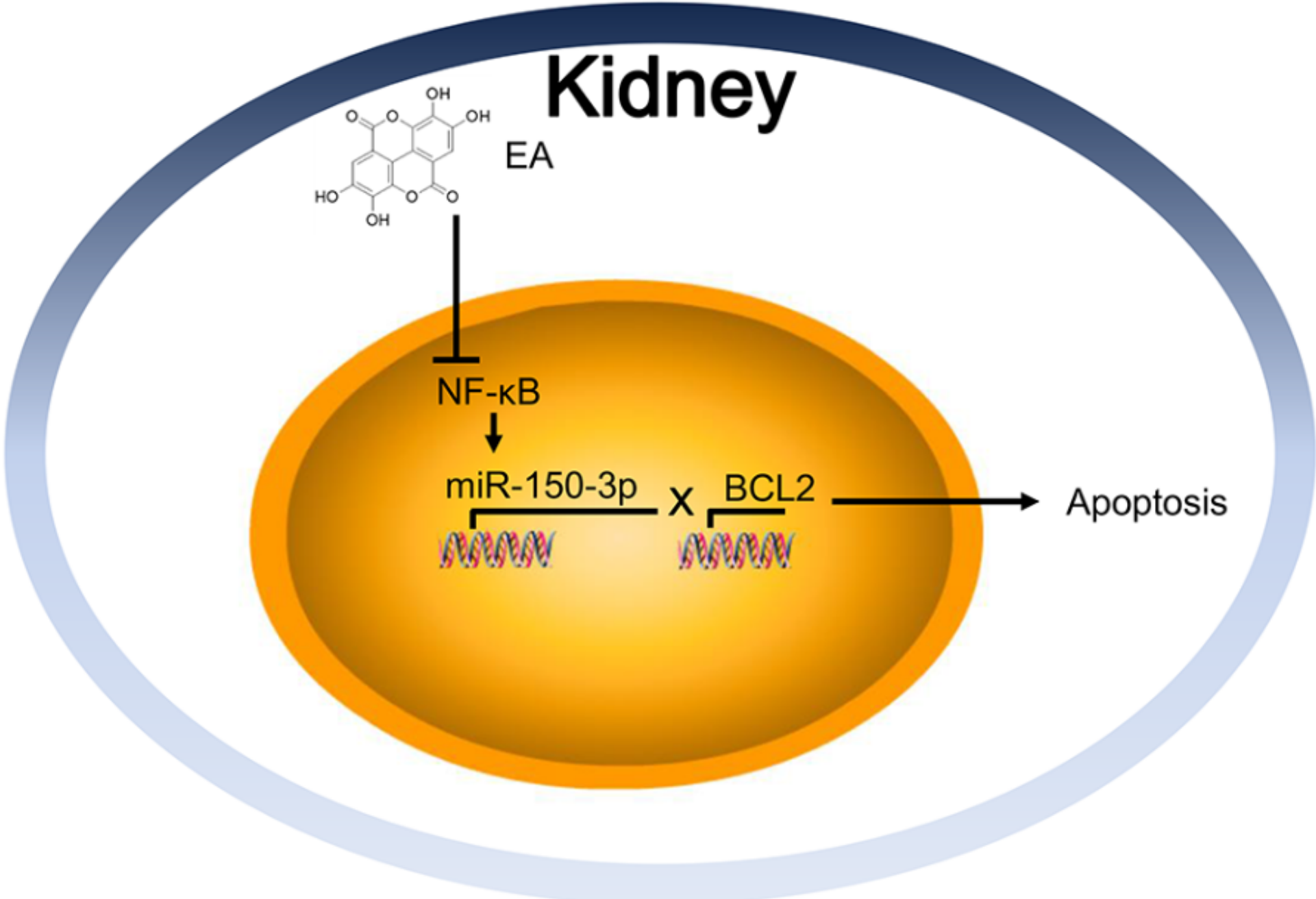

Figure 10

Schematic representation of EA modulated the suppression of NF-KB/miR-150-3p to activate BCL2 that contributed to prevent HG- or hyperglycemia-induced renal dysfunction. 